\title{
A New Class of Fifth and Sixth Order Root-Finding Methods with Its Dynamics and Applications
}

\author{
Debasis Sharma $^{{ }^{*}}$, Sanjaya Kumar Parhi ${ }^{2}$, Shanta Kumari Sunanda ${ }^{1}$ \\ ${ }^{1}$ Department of Mathematics, IIIT Bhubaneswar, Odisha 751003, India \\ ${ }^{2}$ Department of Mathematics, Fakir Mohan University, Odisha 756020, India \\ Email: iiit.debasis@gmail.com
}

\begin{abstract}
In this paper, we deal with the construction, analysis and applications of a modified uniparametric family of methods to solve nonlinear equations in $\mathbb{R}$. We study the convergence of new methods which shows the order of convergence is at least five and for a particular value $\frac{3}{2}$ of the parameter $\gamma$, the method is sixth-order convergent. We discuss several applications such as Max Planck's conservative law, chemical equilibrium, and multi-factor effect to demonstrate the productiveness and capability of the suggested method (for $\gamma=\frac{3}{2}$ ). At every iteration our method is compared with Maroju et al. method ${ }^{[1]}$ and Parhi and Gupta method ${ }^{[2]}$ in terms of the values $\left|f\left(x_{n}\right)\right|$ and $\left|x_{n}-x_{n-1}\right|$. From the numerical experiments, the advantages of our method are observed. Furthermore, we study the complex dynamics to determine the stability and dynamical properties of the methods.

Keywords: iterative methods, nonlinear equations, Newton's method, complex dynamics, stability
\end{abstract}

\section{Introduction}

In the course of recent years, numerous researchers have endeavored to develop higher order convergent iterative schemes to solve nonlinear equations

$$
f(x)=0
$$

where $f: \mathbb{D} \subseteq \mathbb{R} \rightarrow \mathbb{R}$ be a sufficiently differentiable nonlinear function on an open interval $\mathbb{D}$. One of the most frequently used parametric iterative schemes for solving nonlinear equations is Chebyshev-Halley's method. These methods are cubically convergent. Over the past few years, a lot of modifications of Chebyshev-Halley's methods have been studied by numerous authors with enhancement in convergence $\operatorname{order}^{[3-6]}$. They tried to remove the issue of evaluating second order derivative because of the high operational cost. That is why the study of convergence of uni-parametric class of iterative methods free from second derivative has been discussed in [3, 7-8]. In [9], a second derivative free Chebyshev-type methods is studied. The convergence of second derivative free Chebyshev-Halley methods is analyzed in [10] by Li et al. Due to the greater efficiency of computation, the second order Newton's method ${ }^{[11]}$ is also widely used to solve nonlinear equations in $\mathbb{R}$. In addition, construction of multi-point higher order schemes becomes important and more popular due to the improvement in digital computers and symbolic computing. In the most recent decade, numerous researchers around the world have studied multi-point iterative scheme ${ }^{[1-2,12-33]}$ with their convergence and complex dynamical properties.

In this article, a new uni-parametric class of iterative methods is developed by modifying a family of methods, independent of second derivative, proposed by Kou et al. in [33]. The analysis of convergence reveals that our method is fifth order convergent for the parameter $\gamma \in \mathbb{R}$ and sixth order convergent for $\gamma=\frac{3}{2}$. To implement the new methods, we need two evaluations of the function and two of its first derivative per iteration with efficiency index 1.565. Several numerical models such as Max Planck's conservative law, chemical equilibrium and multi-factor effect are worked out to demonstrate that the new iterative scheme is effective and superior than existing methods. For a particular case of its parameter, the complex dynamics is discussed.

Copyright (C2020 Debasis Sharma, et al.

DOI: https://doi.org/10.37256/cm.152020606

This is an open-access article distributed under a CC BY license

(Creative Commons Attribution 4.0 International License)

https://creativecommons.org/licenses/by/4.0/ 


\section{The proposed method and convergence analysis}

In this section, the proposed second derivative free family of methods is described to find a simple root $\alpha$, i.e., $f(\alpha)$ $=0$ and $f^{\prime}(\alpha) \neq 0$ of

$$
f(x)=0,
$$

where $f: \mathbb{R} \rightarrow \mathbb{R}$, be the continuously differentiable function.

Let us consider the family of iterative methods suggested by Kou et al. in [6]:

$$
y_{n}=x_{n}-\frac{2}{3} \frac{f\left(x_{n}\right)}{f^{\prime}\left(x_{n}\right)},
$$

$$
x_{n+1}=x_{n}-\left[1-\frac{3}{4} \frac{\frac{f^{\prime}\left(y_{n}\right)}{f^{\prime}\left(x_{n}\right)}-1}{\gamma \frac{f^{\prime}\left(y_{n}\right)}{f^{\prime}\left(x_{n}\right)}+1-\gamma}\right] \frac{f\left(x_{n}\right)}{f^{\prime}\left(x_{n}\right)}
$$

We want to generalize the above methods by composing Newton's method as follows:

$$
\begin{aligned}
& y_{n}=x_{n}-\frac{2}{3} \frac{f\left(x_{n}\right)}{f^{\prime}\left(x_{n}\right)}, \\
& z_{n}=x_{n}-\left[1-\frac{3}{4} \frac{\frac{f^{\prime}\left(y_{n}\right)}{f^{\prime}\left(x_{n}\right)}-1}{\gamma \frac{f^{\prime}\left(y_{n}\right)}{f^{\prime}\left(x_{n}\right)}+1-\gamma}\right] \frac{f\left(x_{n}\right)}{f^{\prime}\left(x_{n}\right)}, \\
& x_{n+1}=z_{n}-\frac{f\left(z_{n}\right)}{f^{\prime}\left(z_{n}\right)}
\end{aligned}
$$

Taylor's approximation is applied to reduce the number of function evaluations in the above iterative methods. Applying Taylor's expansion on $f^{\prime}\left(z_{n}\right)$ about $z_{n}=x_{n}$ we obtain

$$
f^{\prime}\left(z_{n}\right) \approx f^{\prime}\left(x_{n}\right)+\left(z_{n}-x_{n}\right) f^{\prime \prime}\left(x_{n}\right) .
$$

Using divided difference

$$
f^{\prime \prime}\left(x_{n}\right) \approx \frac{f^{\prime}\left(y_{n}\right)-f^{\prime}\left(x_{n}\right)}{y_{n}-x_{n}} .
$$

Therefore,

$$
f^{\prime}\left(z_{n}\right) \approx f^{\prime}\left(x_{n}\right)+\left(z_{n}-x_{n}\right) \frac{f^{\prime}\left(y_{n}\right)-f^{\prime}\left(x_{n}\right)}{y_{n}-x_{n}} .
$$

Replacing $f^{\prime}\left(z_{n}\right)$ in (3), the proposed family of methods can be described as below. 


$$
\begin{aligned}
& y_{n}=x_{n}-\frac{2}{3} \frac{f\left(x_{n}\right)}{f^{\prime}\left(x_{n}\right)}, \\
& z_{n}=x_{n}-\left[1-\frac{3}{4} \frac{\frac{f^{\prime}\left(y_{n}\right)}{f^{\prime}\left(x_{n}\right)}-1}{\gamma \frac{f^{\prime}\left(y_{n}\right)}{f^{\prime}\left(x_{n}\right)}+1-\gamma}\right] \frac{f\left(x_{n}\right)}{f^{\prime}\left(x_{n}\right)}, \\
& x_{n+1}=z_{n}-\frac{f\left(z_{n}\right)}{f^{\prime}\left(x_{n}\right)+\left(z_{n}-x_{n}\right) \frac{f^{\prime}\left(y_{n}\right)-f^{\prime}\left(x_{n}\right)}{y_{n}-x_{n}}}, n=0,1,2, \ldots,
\end{aligned}
$$

where $\gamma \in \mathbb{R}$ and $x_{0}$ is a suitably chosen initial approximation to the root $\alpha$. Convergence analysis of the new class of methods defined by (4) is established in the next theorem.

\subsection{Convergence analysis}

Theorem 1. Let $f: \mathbb{R} \rightarrow \mathbb{R}$ be a sufficiently differentiable function in $\mathbb{R}$ and $x_{0}$ is close to its simple root $\alpha \in \mathbb{R}$. The family of iterative methods defined by (4) has convergence order at least five with the error equation

$$
e_{n+1}=\frac{2}{3} \frac{c_{2}^{2} c_{3}(2 \gamma-3)}{c_{1}^{3}} e_{n}^{5}+O\left(e_{n}^{6}\right)
$$

Moreover, for $\gamma=\frac{3}{2}$, the convergence order is six with the error equation

$$
e_{n+1}=\frac{1}{27} \frac{27 c_{1} c_{2} c_{3}^{2}-3 c_{1}^{2} c_{3} c_{4}-27 c_{2}^{3} c_{3}}{c_{1}^{4}} e_{n}^{6}+O\left(e_{n}^{7}\right)
$$

where $e_{n}=x_{n}-\alpha$ and $c_{j}=\frac{f^{j}(\alpha)}{j !}$, for $j=1,2,3, \ldots$

Proof. Applying Taylor's expansion on $f\left(x_{n}\right)$ yields

$$
f\left(x_{n}\right)=c_{1} e_{n}+c_{2} e_{n}^{2}+c_{3} e_{n}^{3}+c_{4} e_{n}^{4}+O\left(e_{n}^{5}\right)
$$

Similarly,

$$
f^{\prime}\left(x_{n}\right)=1+2 c_{2} e_{n}+3 c_{3} e_{n}^{2}+4 c_{4} e_{n}^{3}+O\left(e_{n}^{4}\right) \text {. }
$$

Therefore,

$$
\frac{f\left(x_{n}\right)}{f^{\prime}\left(x_{n}\right)}=e_{n}-\frac{c_{2}}{c_{1}} e_{n}^{2}-\frac{2\left(c_{1} c_{3}-c_{2}^{2}\right)}{c_{1}^{2}} e_{n}^{3}-\frac{3 c_{1}^{2} c_{4}-7 c_{1} c_{2} c_{3}+4 c_{2}^{3}}{c_{1}^{3}} e_{n}^{4}+O\left(e_{n}^{5}\right) .
$$

Now,

$$
y_{n}=x_{n}-\frac{2}{3} \frac{f\left(x_{n}\right)}{f^{\prime}\left(x_{n}\right)}=\alpha+\frac{1}{3} e_{n}+\frac{2}{3} \frac{c_{2}}{c_{1}} e_{n}^{2}+\frac{4}{3} \frac{c_{1} c_{3}-c_{2}^{2}}{c_{1}^{2}} e_{n}^{3}+\frac{2}{3} \frac{3 c_{1}^{2} c_{4}-7 c_{1} c_{2} c_{3}+4 c_{2}^{3}}{c_{1}^{3}} e_{n}^{4}+O\left(e_{n}^{5}\right) .
$$


Expanding $f^{\prime}\left(y_{n}\right)$ using Taylor's series about $\alpha$ gives us

$f^{\prime}\left(y_{n}\right)=c_{1}+\frac{2}{3} c_{2} e_{n}+\frac{1}{3} \frac{c_{1} c_{3}+4 c_{2}^{2}}{c_{1}} e_{n}^{2}+\ldots+O\left(e_{n}^{5}\right)$

Now,

$z_{n}=x_{n}-\left[1-\frac{3}{4} \frac{\frac{f^{\prime}\left(y_{n}\right)}{f^{\prime}\left(x_{n}\right)}-1}{\gamma \frac{f^{\prime}\left(y_{n}\right)}{f^{\prime}\left(x_{n}\right)}+1-\gamma}\right] \frac{f\left(x_{n}\right)}{f^{\prime}\left(x_{n}\right)}=\alpha-\frac{2}{3} \frac{c_{2}^{2}(-3+2 \gamma)}{c_{1}^{2}} e_{n}^{3}+\ldots+O\left(e_{n}^{5}\right)$.

Employing Taylor's series on $f\left(z_{n}\right)$ about $\alpha$, we obtain

$f\left(z_{n}\right)=-\frac{2}{3} \frac{c_{2}^{2}(-3+2 \gamma)}{c_{1}} e_{n}^{3}+\ldots+O\left(e_{n}^{5}\right)$

Thus,

$$
\begin{aligned}
x_{n+1}=\alpha+\frac{2}{3} \frac{c_{2}^{2} c_{3}(-3+2 \gamma)}{c_{1}^{3}} e_{n}^{5}+\left[\frac{48\left(\gamma-\frac{3}{2}\right)^{2} c_{2}^{5}+48\left(\gamma^{2}-\frac{15}{4} \gamma+\frac{45}{16}\right) c_{1} c_{2}^{3} c_{3}}{c_{1}^{5}}\right. \\
\left.+\frac{64\left(\gamma-\frac{3}{2}\right) c_{4} c_{1}^{2} c_{2}^{2}+144\left(\gamma-\frac{21}{16}\right) c_{3}^{2} c_{1}^{2} c_{2}-3 c_{1}^{3} c_{3} c_{4}}{c_{1}^{5}}\right] e_{n}^{6}+O\left(e_{n}^{7}\right) .
\end{aligned}
$$

Hence, the error equation is given by

$$
\begin{aligned}
& e_{n+1}=\alpha+\frac{2}{3} \frac{c_{2}^{2} c_{3}(-3+2 \gamma)}{c_{1}^{3}} e_{n}^{5}+\left[\frac{48\left(\gamma-\frac{3}{2}\right)^{2} c_{2}^{5}+48\left(\gamma^{2}-\frac{15}{4} \gamma+\frac{45}{16}\right) c_{1} c_{2}^{3} c_{3}}{c_{1}^{5}}\right. \\
& \left.+\frac{64\left(\gamma-\frac{3}{2}\right) c_{4} c_{1}^{2} c_{2}^{2}+144\left(\gamma-\frac{21}{16}\right) c_{3}^{2} c_{1}^{2} c_{2}-3 c_{1}^{3} c_{3} c_{4}}{c_{1}^{5}}\right] e_{n}^{6}+O\left(e_{n}^{7}\right) .
\end{aligned}
$$

Therefore, the convergence order of iterative scheme is six for $\gamma=\frac{3}{2}$ and five for all other real values of $\gamma$. The error equation for $\gamma=\frac{3}{2}$ is

$$
e_{n+1}=\frac{1}{27} \frac{27 c_{1} c_{2} c_{3}^{2}-3 c_{1}^{2} c_{3} c_{4}-27 c_{2}^{3} c_{3}}{c_{1}^{4}} e_{n}^{6}+O\left(e_{n}^{7}\right)
$$




\section{Numerical applications}

In this section, the productiveness of the proposed method is verified using different test functions. For $\gamma=\frac{3}{2}$ the proposed sixth order iterative scheme is:

$$
\begin{aligned}
& y_{n}=x_{n}-\frac{2}{3} \frac{f\left(x_{n}\right)}{f^{\prime}\left(x_{n}\right)}, \\
& z_{n}=x_{n}-\left[\frac{f^{\prime}\left(x_{n}\right)+3 f^{\prime}\left(y_{n}\right)}{6 f^{\prime}\left(y_{n}\right)-2 f^{\prime}\left(x_{n}\right)}\right] \frac{f\left(x_{n}\right)}{f^{\prime}\left(x_{n}\right)}, \\
& x_{n+1}=z_{n}-\frac{f\left(z_{n}\right)\left[4 f^{\prime}\left(x_{n}\right)-12 f^{\prime}\left(y_{n}\right)\right]}{7 f^{\prime}\left(x_{n}\right)^{2}-6 f^{\prime}\left(x_{n}\right) f^{\prime}\left(y_{n}\right)-9 f^{\prime}\left(y_{n}\right)^{2}}, n=0,1,2, \ldots,
\end{aligned}
$$

Example $1 f_{1}(x)=x^{3}+4 x^{2}-10, x_{0}=1, \alpha=1.3652300134140969$.

Example $2 f_{2}(x)=\sin ^{2} x-x^{2}+1, x_{0}=1, \alpha=1.4044916482153411$.

Example $3 f_{3}(x)=e^{-x}+\cos x, x_{0}=2, x_{0}=1.74613953040801242$.

Example $4 f_{4}(x)=x^{2}-e^{-x}-3 x+2, x_{0}=1, \alpha=0.60898910301016549$.

Example $5 f_{5}(x)=e^{x} \sin x+\log \left(x^{2}+1\right), x_{0}=1, \alpha=0$.

Example 6 We consider the Planck's radiation law problem given in [1]. "This problem deals with the calculation of the energy density within an isothermal blackbody and is given by:

$$
\vartheta(\lambda)=\frac{8 \pi c P \lambda^{-5}}{e^{\frac{c P}{\lambda B T}}-1},
$$

where $\lambda$ is the wavelength of the radiation, $T$ is the absolute temperature of the blackbody, $B$ is the Boltzmann constant, $P$ is the Planck constant and $\mathrm{c}$ is the speed of light." Our interest is to find the wavelength $\lambda$ which maximize the energy density $\vartheta(\lambda)$. From $(8)$, we get

$$
\vartheta^{\prime}(\lambda)=\left(\frac{8 \pi c P \lambda^{-6}}{e^{\frac{c P}{\lambda B T}}-1}\right)\left(\frac{\frac{c P}{\lambda B T} e^{\frac{c P}{\lambda B T}}}{\frac{c P}{e^{\frac{\lambda B T}{\lambda T}}-1}-5}\right)
$$

$\vartheta(\lambda)$ will be maximum when

$$
\frac{\frac{c P}{\lambda B T} e^{\frac{c P}{\lambda B T}}}{e^{\frac{c P}{\lambda B T}}-1}=5 .
$$

By substituting $x=\frac{c P}{\lambda B T}$, the equation (10) is satisfied if

$$
f_{6}(x)=e^{-x}+\frac{x}{5}-1=0 .
$$


Thus, the solutions of $f_{6}(x)=0$ produce the maximum wavelength of radiation $\lambda$ by means of the following formula:

$$
\lambda \approx \frac{c P}{\alpha B T}
$$

where $\alpha$ is a solution of $f_{6}(x)=0$. As function $f_{6}(x)$ is always continuous and satisfies $f_{6}(1)=-0.432121 \ldots$ and $f_{6}(8.5)=$ $0.700203 \ldots$, clearly $f_{6}(x)$ has zeros in the interval [1]. So, we choose $D=[1, \infty)$ and $\alpha=4.965114$.

Example 7 "This problem [32] involves a single nonlinear equation, studied by Shacham (1989), which represents the chemical equilibrium of the $\mathrm{N}_{2}-\mathrm{H}_{2}-\mathrm{NH}_{3}$ system at $500^{\circ} \mathrm{C}$ and $250 \mathrm{~atm}$, where the variable, $x$, is the fractional conversion of nitrogen starting with a stoichiometric mixture of $\mathrm{N}_{2}$ and $\mathrm{H}_{2}$. The equation is given by

$$
f_{7}(x)=\frac{8(4-x)^{2} x^{2}}{(6-3 x)^{2}(2-x)}-0.186=0, x_{0}=1.25, \alpha=0.277759542 .
$$

Example 8 We solve the following problem given in [1].

"In the study of the multi-factor effect, the trajectory of an electron in the air gap between two parallel plates is given by

$$
x(t)=x_{0}+\left(v_{0}+e \frac{E_{0}}{m w} \sin \left(w t_{0}+\theta\right)\right)\left(t-t_{0}\right)+e \frac{E_{0}}{m w^{2}}(\cos (w t+\theta)+\sin (w t+\theta))
$$

where $e$ and $m$ are the charge and the mass of the electron at rest, $x_{0}$ and $v_{0}$ are the position and velocity of the electron at time $t_{0}$ and $E_{0} \sin (w t+\theta)$ is the RF electric field between the plates. We choose the particulars parameters in the expression in order to deal with a simpler expression, which is defined as follows:

$$
f_{8}(x)=x-\frac{1}{2} \cos x+\frac{\pi}{4}, \alpha=-0.3090932715417949 \text {. }
$$


Table 1. Comparison of iterative methods for the test functions

\begin{tabular}{|c|c|c|c|c|c|c|c|}
\hline \multirow{2}{*}{$f(x)$} & \multirow{2}{*}{$n$} & \multicolumn{2}{|c|}{$P G M^{[2]}$} & \multicolumn{2}{|c|}{ Maroju et al..$^{[1]}$} & \multicolumn{2}{|c|}{$P M$} \\
\hline & & $\left|f\left(x_{n}\right)\right|$ & $\left|x_{n}-x_{n-1}\right|$ & $\left|f\left(x_{n}\right)\right|$ & $\left|x_{n}-x_{n-1}\right|$ & $\left|f\left(x_{n}\right)\right|$ & $\left|x_{n}-x_{n-1}\right|$ \\
\hline \multirow[t]{4}{*}{$f_{1}(x)$} & 1 & $1.01 \times 10^{-3}$ & $3.65 \times 10^{-1}$ & $5.35 \times 10^{-4}$ & $3.65 \times 10^{-1}$ & $1.34 \times 10^{-4}$ & $3.65 \times 10^{-1}$ \\
\hline & 2 & $1.03 \times 10^{-26}$ & $6.12 \times 10^{-5}$ & $3.78 \times 10^{-28}$ & $3.24 \times 10^{-5}$ & $2.49 \times 10^{-32}$ & $8.10 \times 10^{-6}$ \\
\hline & 3 & $1.13 \times 10^{-164}$ & $6.22 \times 10^{-28}$ & $4.66 \times 10^{-173}$ & $2.29 \times 10^{-29}$ & $1.04 \times 10^{-198}$ & $1.51 \times 10^{-33}$ \\
\hline & 4 & $2.00 \times 10^{-992}$ & $6.84 \times 10^{-166}$ & $1.63 \times 10^{-1042}$ & $2.82 \times 10^{-174}$ & $5.68 \times 10^{-1197}$ & $6.33 \times 10^{-200}$ \\
\hline \multirow[t]{4}{*}{$f_{2}(x)$} & 1 & $8.18 \times 10^{-3}$ & $4.08 \times 10^{-1}$ & $7.1 \times 10^{-2}$ & $4.33 \times 10^{-1}$ & $1.3 \times 10^{-3}$ & $4.05 \times 10^{-1}$ \\
\hline & 2 & $6.28 \times 10^{-16}$ & $3.3 \times 10^{-3}$ & $1.24 \times 10^{-10}$ & $2.80 \times 10^{-2}$ & $1.73 \times 10^{-21}$ & $5.21 \times 10^{-4}$ \\
\hline & 3 & $1.33 \times 10^{-94}$ & $2.53 \times 10^{-16}$ & $4.77 \times 10^{-63}$ & $5.02 \times 10^{-11}$ & $9.83 \times 10^{-129}$ & $6.96 \times 10^{-22}$ \\
\hline & 4 & $1.18 \times 10^{-566}$ & $5.35 \times 10^{-95}$ & $1.50 \times 10^{-377}$ & $1.92 \times 10^{-63}$ & $3.33 \times 10^{-772}$ & $3.96 \times 10^{-129}$ \\
\hline \multirow[t]{4}{*}{$f_{3}(x)$} & 1 & $5.65 \times 10^{-7}$ & $2.54 \times 10^{-1}$ & $1.99 \times 10^{-6}$ & $2.54 \times 10^{-1}$ & $1.16 \times 10^{-6}$ & $2.54 \times 10^{-1}$ \\
\hline & 2 & $2.61 \times 10^{-41}$ & $4.87 \times 10^{-7}$ & $9.62 \times 10^{-38}$ & $1.72 \times 10^{-6}$ & $2.82 \times 10^{-39}$ & $9.99 \times 10^{-7}$ \\
\hline & 3 & $2.52 \times 10^{-247}$ & $2.25 \times 10^{-41}$ & $1.22 \times 10^{-225}$ & $8.3 \times 10^{-38}$ & $5.82 \times 10^{-235}$ & $2.43 \times 10^{-39}$ \\
\hline & 4 & $2.06 \times 10^{-1483}$ & $2.17 \times 10^{-247}$ & $5.20 \times 10^{-1353}$ & $1.05 \times 10^{-225}$ & $4.52 \times 10^{-1409}$ & $5.02 \times 10^{-235}$ \\
\hline \multirow[t]{4}{*}{$f_{4}(x)$} & 1 & $2.8 \times 10^{-3}$ & $3.9 \times 10^{-1}$ & $1.07 \times 10^{-2}$ & $4.0 \times 10^{-1}$ & $3.22 \times 10^{-4}$ & $3.9 \times 10^{-1}$ \\
\hline & 2 & $1.52 \times 10^{-17}$ & $2.25 \times 10^{-3}$ & $2.32 \times 10^{-14}$ & $8.5 \times 10^{-3}$ & $6.88 \times 10^{-24}$ & $2.6 \times 10^{-4}$ \\
\hline & 3 & $4.09 \times 10^{-103}$ & $1.23 \times 10^{-17}$ & $2.58 \times 10^{-84}$ & $1.88 \times 10^{-14}$ & $6.52 \times 10^{-142}$ & $5.55 \times 10^{-24}$ \\
\hline & 4 & $1.54 \times 10^{-616}$ & $3.30 \times 10^{-103}$ & $4.8 \times 10^{-504}$ & $2.08 \times 10^{-84}$ & $4.72 \times 10^{-850}$ & $5.27 \times 10^{-142}$ \\
\hline \multirow[t]{4}{*}{$f_{5}(x)$} & 1 & $5.2 \times 10^{-2}$ & $9.52 \times 10^{-1}$ & $5.34 \times 10^{-2}$ & $9.51 \times 10^{-1}$ & $4.06 \times 10^{-2}$ & $9.62 \times 10^{-1}$ \\
\hline & 2 & $1.98 \times 10^{-7}$ & $4.8 \times 10^{-2}$ & $4.9 \times 10^{-8}$ & $4.86 \times 10^{-2}$ & $5.28 \times 10^{-9}$ & $3.78 \times 10^{-2}$ \\
\hline & 3 & $1.56 \times 10^{-39}$ & $1.98 \times 10^{-7}$ & $1.07 \times 10^{-43}$ & $4.9 \times 10^{-8}$ & $5.24 \times 10^{-50}$ & $5.28 \times 10^{-9}$ \\
\hline & 4 & $3.77 \times 10^{-232}$ & $1.56 \times 10^{-39}$ & $1.17 \times 10^{-257}$ & $1.07 \times 10^{-43}$ & $5.0 \times 10^{-296}$ & $5.24 \times 10^{-50}$ \\
\hline \multirow[t]{4}{*}{$f_{6}(x)$} & 1 & $5.13 \times 10^{-2}$ & 2.69773 & $8.23 \times 10^{-1}$ & 7.114220 & $1.01 \times 10^{-1}$ & 2.912960 \\
\hline & 2 & $7.83 \times 10^{-11}$ & $2.67 \times 10^{-1}$ & $2.31 \times 10^{-6}$ & 4.149094 & $7.05 \times 10^{-15}$ & $5.21 \times 10^{-2}$ \\
\hline & 3 & $6.42 \times 10^{-64}$ & $4.05 \times 10^{-10}$ & $4.3 \times 10^{-37}$ & $1.2 \times 10^{-5}$ & $7.79 \times 10^{-88}$ & $3.65 \times 10^{-14}$ \\
\hline & 4 & $1.94 \times 10^{-382}$ & $3.32 \times 10^{-63}$ & $1.8 \times 10^{-221}$ & $2.23 \times 10^{-36}$ & $1.42 \times 10^{-525}$ & $4.03 \times 10^{-87}$ \\
\hline \multirow[t]{5}{*}{$f_{7}(x)$} & 1 & Divergent & - & Divergent & - & $22.8 \times 10^{-1}$ & $5.20 \times 10^{-1}$ \\
\hline & 2 & Divergent & - & Divergent & - & $2.74 \times 10^{-1}$ & $3.26 \times 10^{-1}$ \\
\hline & 3 & Divergent & - & Divergent & - & $2.08 \times 10^{-4}$ & $1.26 \times 10^{-1}$ \\
\hline & 4 & Divergent & - & Divergent & - & $3.16 \times 10^{-22}$ & $1.33 \times 10^{-4}$ \\
\hline & 5 & Divergent & - & Divergent & - & $3.84 \times 10^{-129}$ & $2.02 \times 10^{-22}$ \\
\hline \multirow[t]{4}{*}{$f_{8}(x)$} & 1 & $1.09 \times 10^{-4}$ & $8.09 \times 10^{-1}$ & $9.30 \times 10^{-5}$ & $8.09 \times 10^{-1}$ & $2.83 \times 10^{-5}$ & $8.09 \times 10^{-1}$ \\
\hline & 2 & $4.23 \times 10^{-28}$ & $1.23 \times 10^{-4}$ & $2.56 \times 10^{-27}$ & $1.1 \times 10^{-4}$ & $3.91 \times 10^{-31}$ & $3.3 \times 10^{-5}$ \\
\hline & 3 & $1.42 \times 10^{-168}$ & $4.98 \times 10^{-28}$ & $1.13 \times 10^{-162}$ & $3.02 \times 10^{-27}$ & $2.73 \times 10^{-186}$ & $4.61 \times 10^{-31}$ \\
\hline & 4 & $2.07 \times 10^{-1011}$ & $1.68 \times 10^{-168}$ & $8.13 \times 10^{-975}$ & $1.33 \times 10^{-162}$ & $3.14 \times 10^{-1117}$ & $3.22 \times 10^{-186}$ \\
\hline
\end{tabular}

\section{Complex dynamical study of a special case of the method}

We use the techniques discussed in $[17,23]$ to investigate the complex dynamical characteristics of the proposed family of fifth and sixth order methods (4) on a polynomial $p(z): \mathbb{C} \rightarrow \mathbb{C}$ of degree two, that is $p(z)=(z-A)(z-B)$. We apply the schemes (4) on $p(z)=(z-A)(z-B), A \neq B$, and use the Möbius map $M(z)=\frac{z-A}{z-B}$, which has the following properties

i) $M(A)=0$,

ii) $M(B)=\infty$ and

iii) $M(\infty)=1$,

to yield the rational map related to the family of schemes (4), whose expression is as follows: 


$$
K_{p}(z, \gamma)=z^{6} \frac{(-3 z+4 \gamma-6)^{2}}{(4 \gamma z-6 z-3)^{2}}, \gamma \in \mathbb{C}
$$

Next, we provide the detailed study of all fixed points of $K_{p}(z, \gamma)$ by explaining their stability.

\subsection{Study of fixed points and their stability}

The fixed points $z=0$ and $z=\infty$ of the rational function $K_{p}(z, \gamma)$ are related to $A$ and $B$ respectively, and therefore these are superattracting fixed points. Further to this, the strange fixed points of the operator $K_{p}(z, \gamma)$ are:

1. $z=1$ and

2. The roots of the polynomial $9 z^{6}+(45-24 \gamma) z^{5}+\left(16 \gamma^{2}-72 \gamma+81\right) z^{4}+\left(16 \gamma^{2}-72 \gamma+81\right) z^{3}+\left(16 \gamma^{2}-72 \gamma+81\right) z^{2}+(45$ $-24 \gamma) z+9$ denoted by $e x_{j}(\gamma), j=1,2,3,4,5,6$.

Figure 1. demonstrates the behavior of these fixed points for real values of $e x_{j}(\gamma), j=1,2,3,4,5,6$. The next result describes some relations among the strange fixed points of $K_{p}(z, \gamma)$.

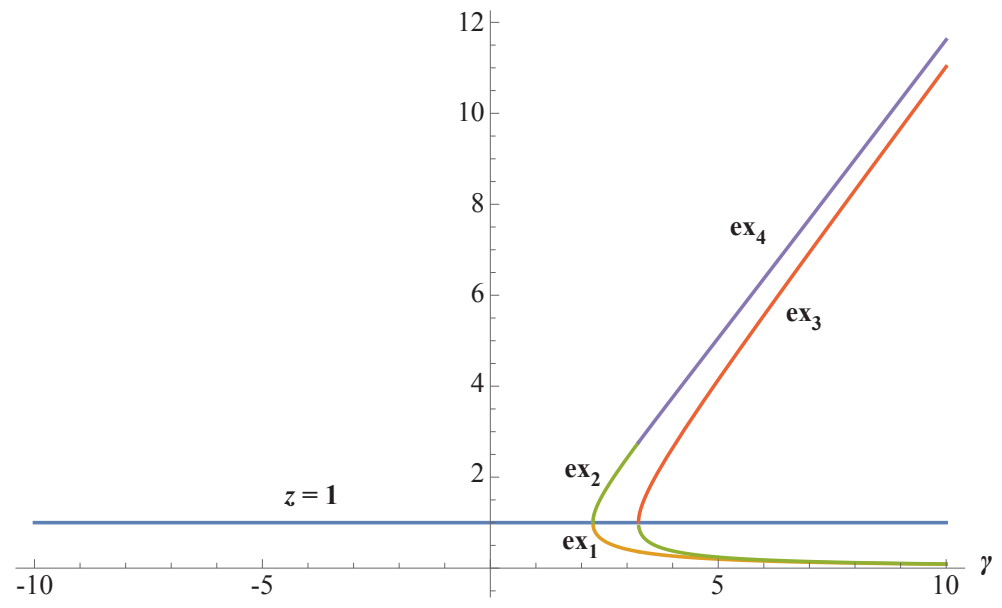

Figure 1. Bifurcation diagram for extraneous fixed points

Lemma 1. The fixed point operator $K_{p}(z, \gamma)$ has seven simple strange fixed points except the following cases:

i) If $\theta=\frac{3}{4}$, then $K_{p}\left(z, \frac{3}{4}\right)=z^{6}$ and hence the family has five strange fixed points.

ii) If $\theta=\frac{9}{4}$, then $K_{p}\left(z, \frac{9}{4}\right)=z^{6}$ and the number of strange fixed points is five.

In order to study the stability of the strange fixed points, we need the first derivative of $K_{p}(z, \gamma)$. Now,

$K_{p}^{\prime}(z, \gamma)=216 z^{5} \frac{\left(\left(\gamma-\frac{3}{2}\right) z^{2}+\left(-\frac{8}{9} \gamma^{2}+\frac{8}{3} \gamma-3\right) z+\gamma-\frac{3}{2}\right)\left(z-\frac{4}{3} \gamma+2\right)}{(4 \gamma z-6 z-3)^{3}}$,

From equation (17), It is obvious that the fixed points 0 and infinity are superattractive in nature for all values of $\gamma$. The stability of $z=1$ and $z=e_{j}(\gamma), j=1,2,3,4,5,6$ will be presented in another way. First of all the stability of the strange fixed point $z=1$ will be analyzed. For the fixed point $z=1$, the stability function is defined by $\left|K_{p}^{\prime}(1, \gamma)\right|$, and denoted by $S t_{1}(\gamma)$.

Theorem 2 The strange fixed point $z=1$ is characterized by

1. The fixed point $z=1$ is superattracting fixed point for $\gamma=3$.

2. The point $z=1$ is an attractive fixed point for $\left|\gamma-\frac{61}{20}\right|<\frac{1}{5}$.

3. If $\left|\gamma-\frac{61}{20}\right|=\frac{1}{5}$, then $z=1$ is a parabolic point.

4. At last, $z=1$ is a repulsor for $\left|\gamma-\frac{61}{20}\right|>\frac{1}{5}$.

Proof. Using equation (17) we get 
$S t_{1}(\gamma)=\left|\frac{16 \gamma-48}{4 \gamma-9}\right|$

It is easy to verify that $S t_{1}(3)=0$.

Again, $\left|\frac{16 \gamma-48}{4 \gamma-9}\right| \leq 1$ is equivalent to $|16 \gamma-48| \leq|4 \gamma-9|$. Let $\gamma=x+i y$ be an arbitrary complex number.

Then,

$256\left(x^{2}-6 x+y^{2}+9\right) \leq 16 x^{2}-72 x+16 y^{2}+81$.

By simplifying

$240 x^{2}+240 y^{2}-1464 x+2223 \leq 0$,

which is equivalent to

$\left(x-\frac{61}{20}\right)^{2}+y^{2} \leq \frac{1}{25}$

Thus, $\left|K_{p}^{\prime}(1, \gamma)\right| \leq 1$ if and only if $\left|\gamma-\frac{61}{20}\right| \leq \frac{1}{5}$.

The region of stability of $z=1$ is the part of $\mathbb{C}$ where $\left|\gamma-\frac{61}{20}\right| \leq \frac{1}{5}$. This region is provided graphically in Figure 2 . It is generally impossible to analytically characterize the stability of the strange points $e x_{j}, j=1,2, \ldots, 6$. However, using mathematica the stability regions of $e x_{j}(j=1,2, \ldots, 6)$ can be studied graphically. These regions are presented in Figures. 3(a)-3(f).

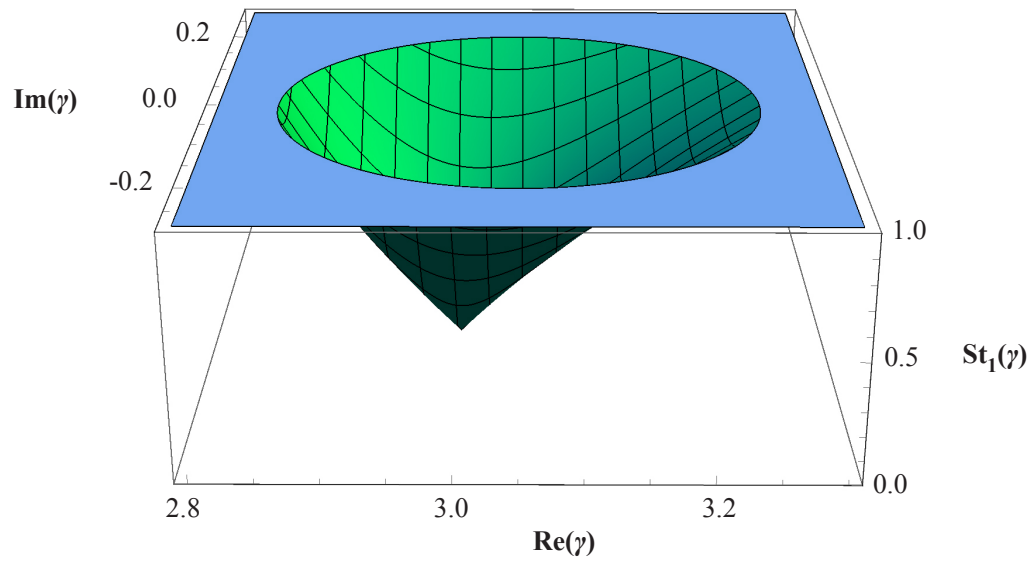

Figure 2. Stability region of $z=1$

Next, we are going to explore the dynamical behaviors of the family by presenting the parameter spaces and dynamical planes related to the family.

\subsection{Study of critical points and parameter spaces}

In this section, we discuss all critical points of the proposed class and the parameter plane associated with free critical points. The solutions of $K_{p}^{\prime}(z, \gamma)=0$ are defined to be the critical points of $K_{p}(z, \gamma)$. From equation (17), we deduce that 0 and $\infty$ are critical points related to $A$ and $B$, and they create the Fatou component of their own. The free critical points of $K_{p}(z)$ are $z=-1$ and the roots of $(\theta+1) z^{4}+4 z^{3}+6 z^{2}+4 z+\theta+1$, and let us write them as $c r_{j}(\gamma), j=1,2,3$. The points $c r_{j}(\gamma)$ are represented as: 
1. $c r_{1}(\gamma)=\frac{4}{3} \gamma-2$

2. $c r_{2}(\gamma)=\frac{1}{9} \frac{8 \gamma^{2}-24 \gamma+27+2 \sqrt{16 \gamma^{4}-96 \gamma^{3}+171 \gamma^{2}-81 \gamma}}{2 \gamma-3}$

3. $c r_{3}(\gamma)=\frac{1}{9} \frac{8 \gamma^{2}-24 \gamma+27-2 \sqrt{16 \gamma^{4}-96 \gamma^{3}+171 \gamma^{2}-81 \gamma}}{2 \gamma-3}$

The following lemma explains the relationship among these free critical points.

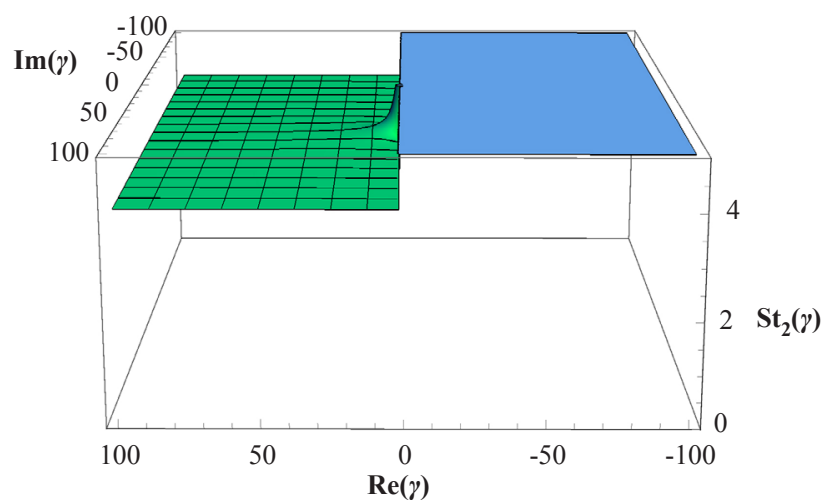

(a) Stability function of $e x_{1}(\gamma)$

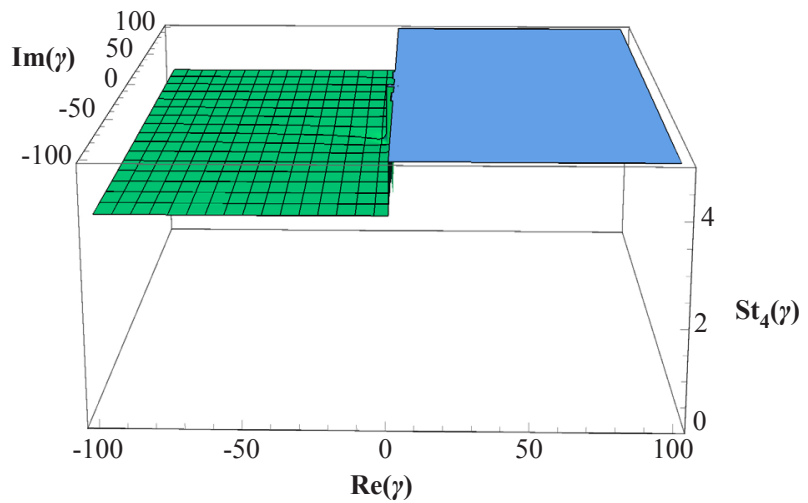

(c) Stability function for $e x_{3}(\gamma)$

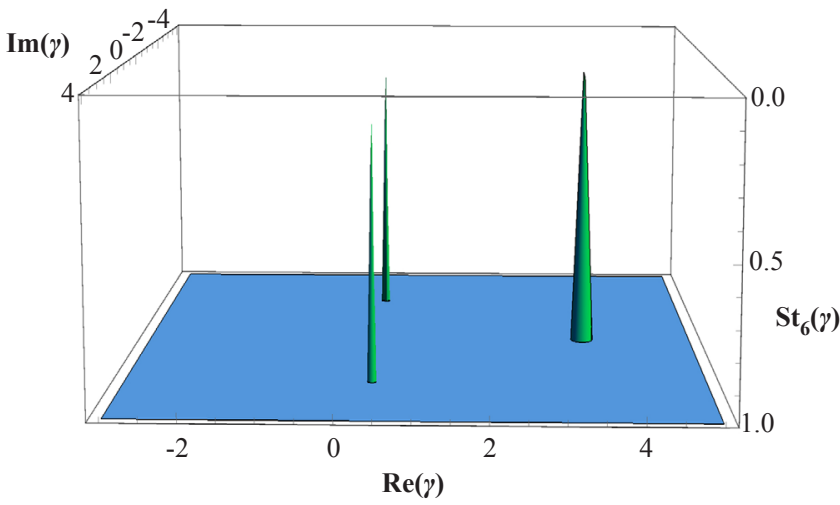

(e) Stability region for $e x_{5}(\gamma)$

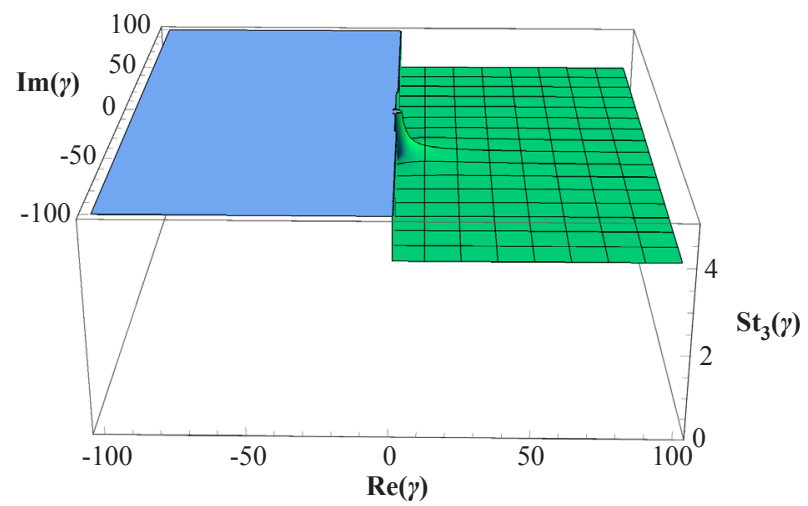

(b) Stability function of $e x_{2}(\gamma)$

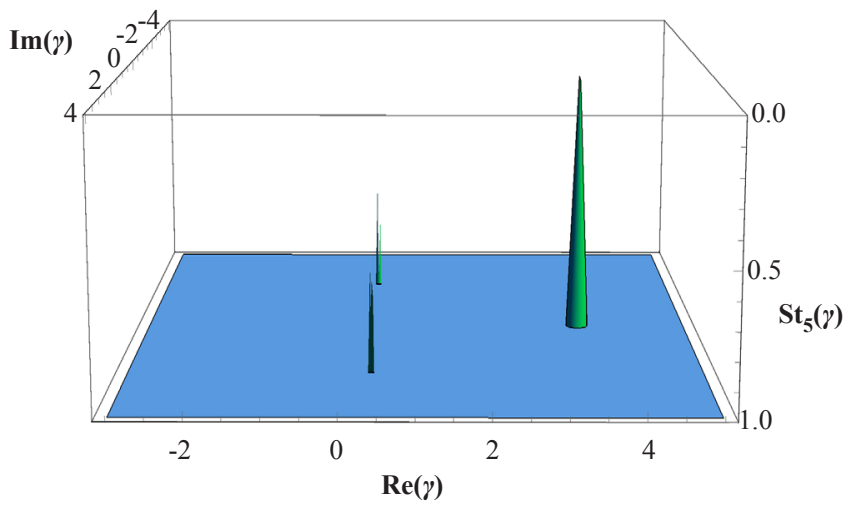

(d) Stability region for $\operatorname{ex}_{4}(\gamma)$

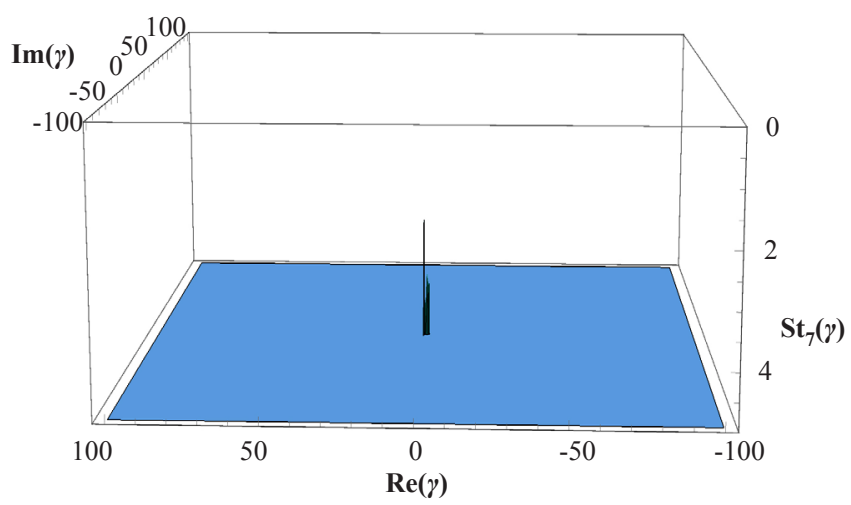

(f) Stability function for $\operatorname{ex}_{6}(\gamma)$

Figure 3. Stability functions 
Lemma 2. i) If $\theta=\frac{3}{4}$, then $c r_{1}=c r_{2}=c r_{3}=-1$. Therefore the number of distinct free critical point is one.

ii) If $\theta=\frac{9}{4}$, then $c r_{1}=c r_{2}=c r_{3}=1$, as a consequence there exists only one free critical point of the family.

iii) If $\theta=0$, then $c r_{2}=c r_{3}=-1$ and hence there exist two different free critical points.

iv) If $\theta=3$, then $c r_{2}=c r_{3}=1$, so the number of distinct free critical points decreases to two.

v) In rest of the cases the family has three distinct free critical points.

It is observed that $c r_{2}(\gamma)=\frac{1}{c r_{3}(\gamma)}$ and therefore, the free critical points $c r_{2}(\gamma)$ and $c r_{3}(\gamma)$ are not independent. Hence, the parameter space related to the free critical point $\mathrm{cr}_{3}(\gamma)$ is presented. Figure 4 shows the bifurcation diagram of these free critical points.

We analyze the behavior of the new family (4) in the complex plane by implementing the complex dynamics technique used in $[17,23]$. The parameter space associated with $c r_{3}(\gamma)$ is given in Figure 5 . By choosing $z_{0}=c r_{j}(\gamma)$ as an initial approximation for the iterative schemes of the proposed family, we assign various colors to $z_{0}$ according to the convergence of the iteration. Cyan represents the convergence of the iteration to 0 (related to A) and magenta denotes that the iteration converges to $\infty$ (related to the B). We paint the initial point $z_{0}$ in yellow for the convergence to 1 (related to $\infty)$. We use maximum 2000 iterations with error tolerance $10^{-6}$. In addition, we use red color to show those point whose corresponding iteration converges to $\operatorname{ex}_{j}(\gamma),(j=1,2,3,4,5,6)$. Other colors such as orange, light green, blue, dark green, dark orange, dark red and white are used to represent the convergence to various $n$-periodic orbits for $n=2,3, \ldots, 8$ respectively. The points painted in black demonstrate the convergence to other $n$-cycles for $n \geq 9$. Thus, the points on the plane presented in different colors other than cyan or magenta are not worthy options for $\gamma$ in terms of better numerical properties of the family.

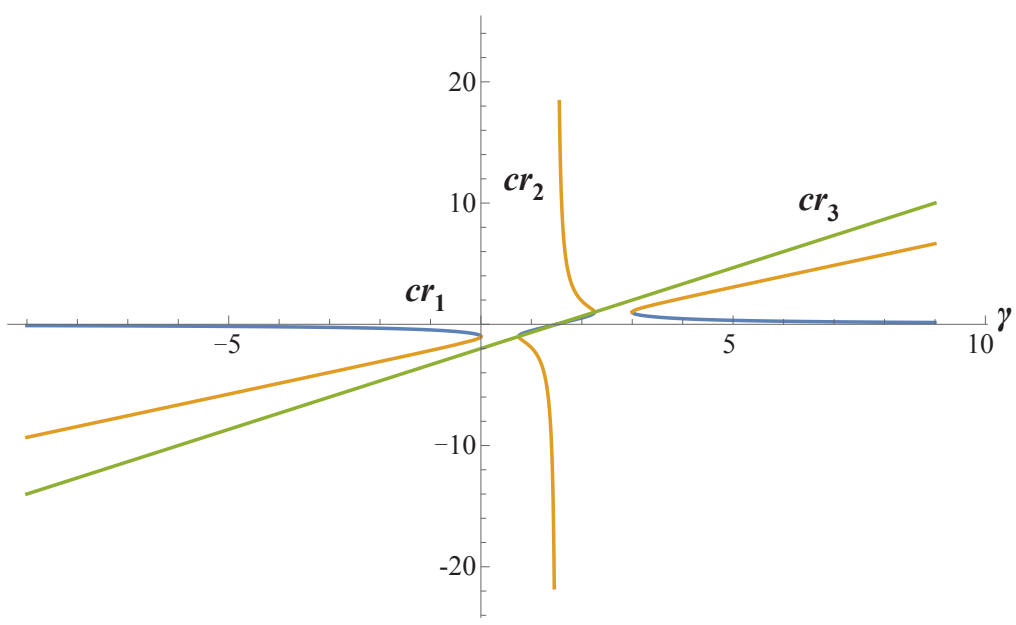

Figure 4. Bifurcation diagram for free critical points

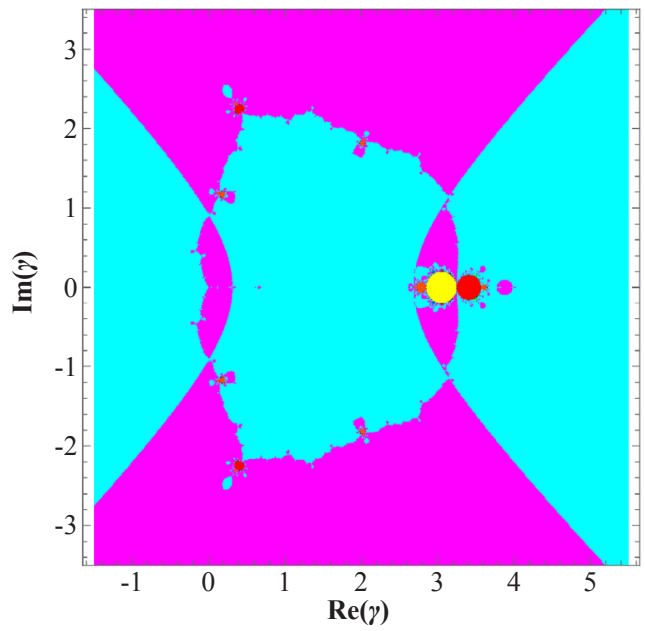

Figure 5. Parameter space associated to the free critical point $c r_{3}(\gamma)$ 
Some anomalies are presented in the dynamical planes. Convergence to 0 appears in cyan and in magenta convergence to $\infty$ is presented in the figures of dynamical plane. Black color specifies the nonconvergence regions. We choose 1000 iterations as the maximum limit and a tolerance of $10^{-6}$. In the dynamical planes shown in Figures 7(a)-7(c), the appearance of the strange fixed point $z=1$ is painted in yellow. In Figures 7(d) and 7(e), convergence towards attracting strange fixed points are presented. The dynamical planes associated with the members of the family for $\gamma=2.8$ and $\gamma=3.6$ with convergence to attracting 2-cycles are presented in Figures 7(f) and 7(g) respectively.

In Figures 7(h) and 8(a), convergence to 3-cycles is painted in black color as there is no convergence to $A$ or $B$. Furthermore, attracting 4-cycles can be seen in the dynamical planes shown in Figures 8(b)-8(c).
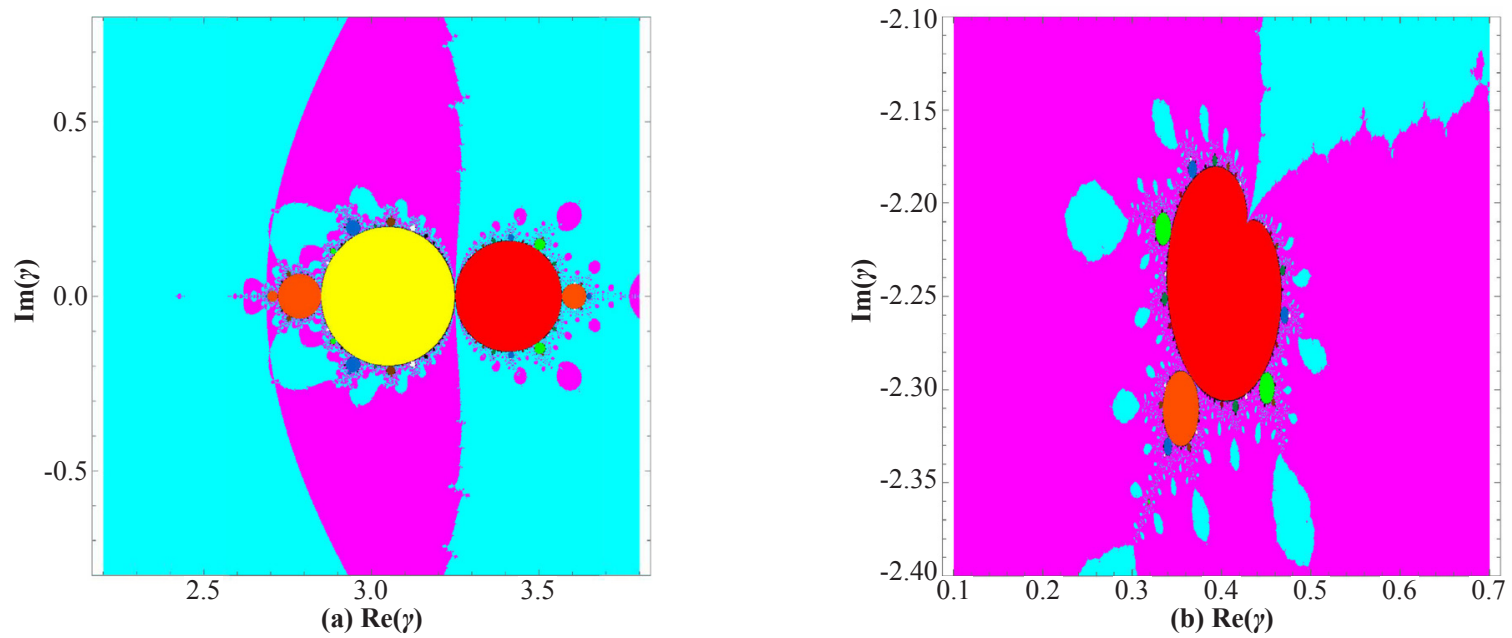

Figure 6. Details of the parameter space associated to the free critical point $c r_{3}(\gamma)$

At the end, we present the dynamical planes for $\gamma=1.5, \gamma=0.5, \gamma=2.5, \gamma=3.9$ and $\gamma=3.7$ in Figures 8(d)-8(h) respectively, where the new family has good convergence properties. In these figures, the attraction basins only represent the convergence related to $A$ or $B$. Consequently, the proposed family shows its high stability thereat in terms of numerical behavior.

We studied the complex dynamics of the family (4) applied to arbitrary second degree polynomial. The stable members of the family are also derived from this study. In order to show some limitations of this study, we test the behavior of some stable members of the family to solve other nonlinear equations. We consider the nonlinear equation $x^{3}+4 x^{2}-10$ $=0$. We apply the stable members of the family (for $\gamma=3.7$ and $\gamma=0.5$ ) to solve this equation with initial point $x_{0}=-2$. The method (for $\gamma=3.7$ ) diverges and the other method (for $\gamma=0.5$ ) converges to the solution $\alpha=1.3652300134140969$ in 17 iterations with tolerance error $10^{-20}$ and maximum iteration 100. However, the poor members of the family (for $\gamma=3.06$ and $\gamma=3.2$ ) converge to the solution in 14 iterations and show better performance. Similarly, we consider another equation $e^{x}-1.5-\arctan (x)=0$. We apply two stable members of the family (for $\gamma=3.7$ and $\gamma=2.5$ ) to solve this equation with initial point $x_{0}=-2$. Both methods (for $\gamma=3.7$ and $\gamma=2.5$ ) show divergent behavior. However, the poor members of the family (for $\gamma=3.06$ and $\gamma=3.2$ ) converge to the solution $\alpha=-14.1012697727399684$ in 8 and 9 iterations respectively with tolerance error $10^{-20}$ and maximum iteration 100 .

The above examples show that some stable members of the family (4) derived from the study of complex dynamics are not effective in all cases. This is a major limitation of complex dynamics of the family applied to quadratic polynomials. From this study, we can only get an overall idea about the behavior of the members of the family. There is no guarantee that the stable members will provide better results for other nonlinear equations in comparison with the poor members.

\section{Conclusion}

We developed a new class of iterative methods to obtain solution of nonlinear equations and studied its convergence analysis. The convergence order of the family of methods is at least five for $\gamma \in \mathbb{R}$ and six for $\gamma=\frac{3}{2}$. Several numerical models are worked out and computed $\left|f\left(x_{n}\right)\right|,\left|x_{n}-x_{n-1}\right|$ with our method, Parhi and Gupta method (PGM) and Maroju et al. method. At last, it is concluded that our method performs much better compared to these methods. In addition, we studied 
the complex dynamics of the class of iterative schemes by characterizing the stability of fixed points and analyzing the parameter planes.

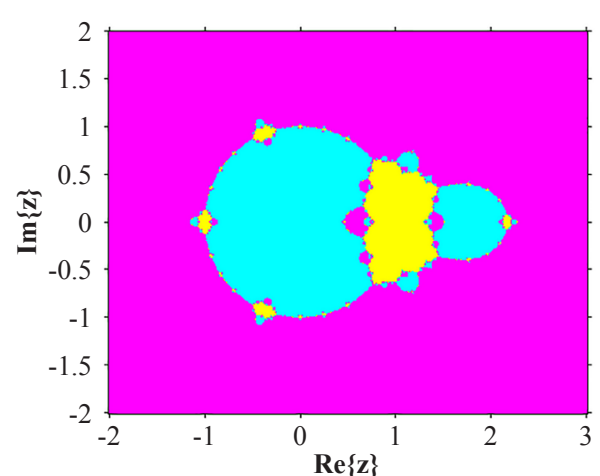

(a) $\gamma=2.9$

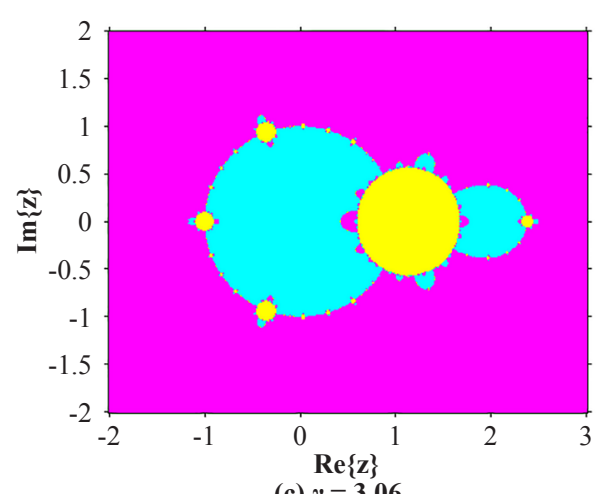

(c) $\gamma=3.06$

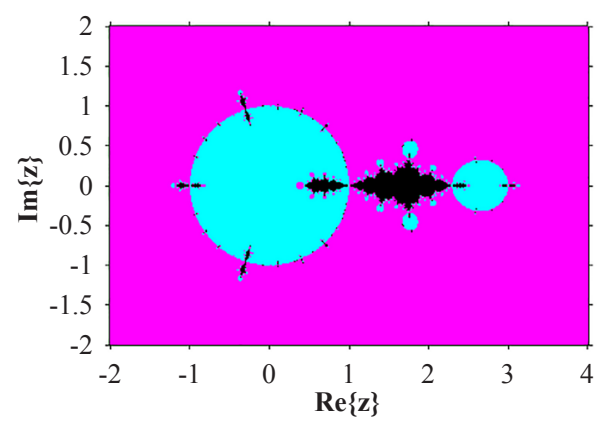

(e) $\gamma=3.55$

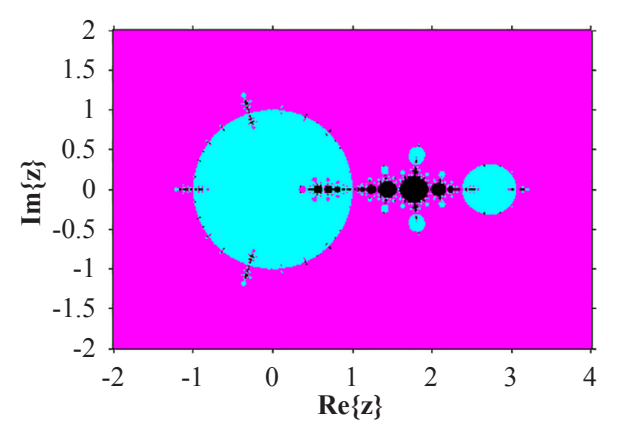

(g) $\gamma=3.6$

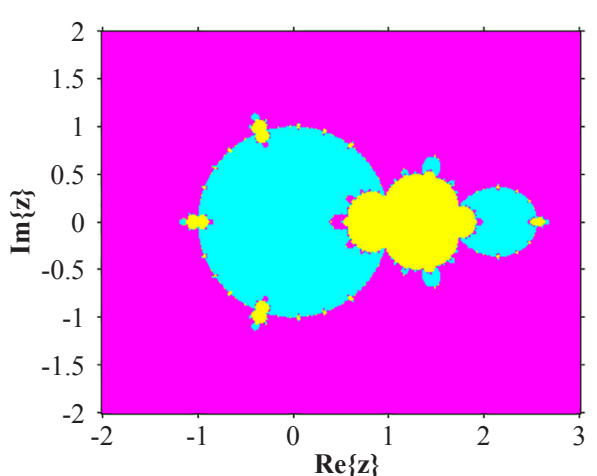

(b) $\gamma=3.2$
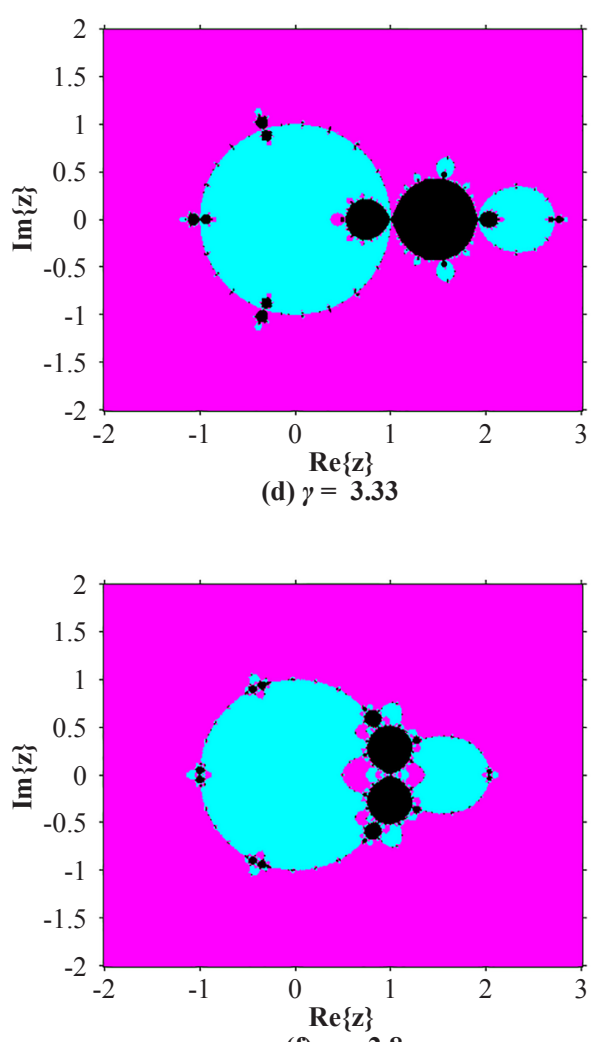

(f) $\gamma=2.8$

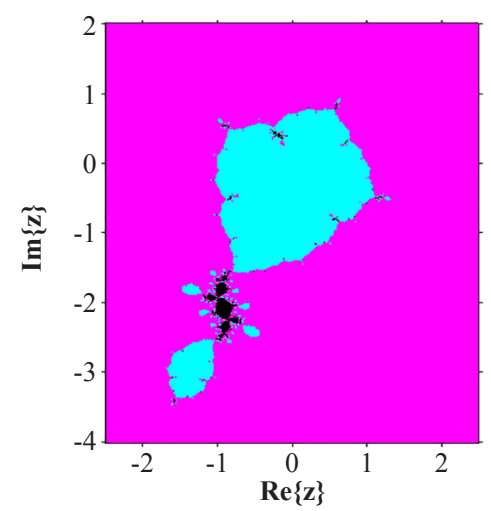

(h) $\gamma=0.45+2.3 i$

Figure 7. Dynamical planes 


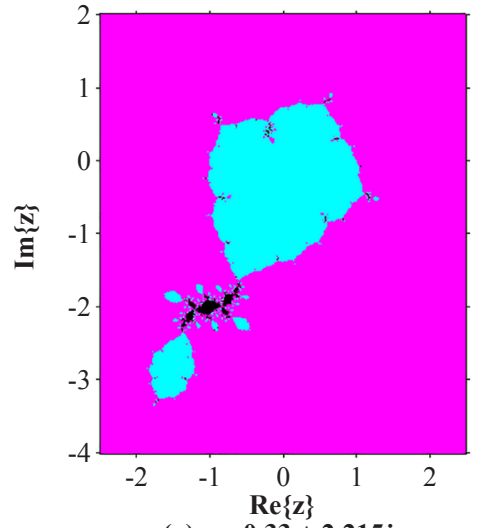

(a) $\gamma=0.33+2.215 i$

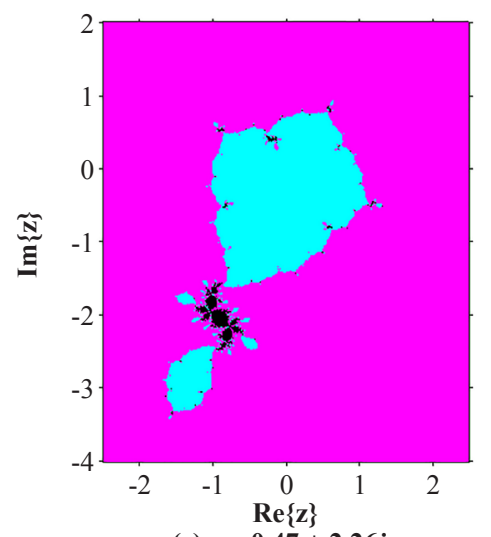

(c) $\gamma=0.47+2.26 i$

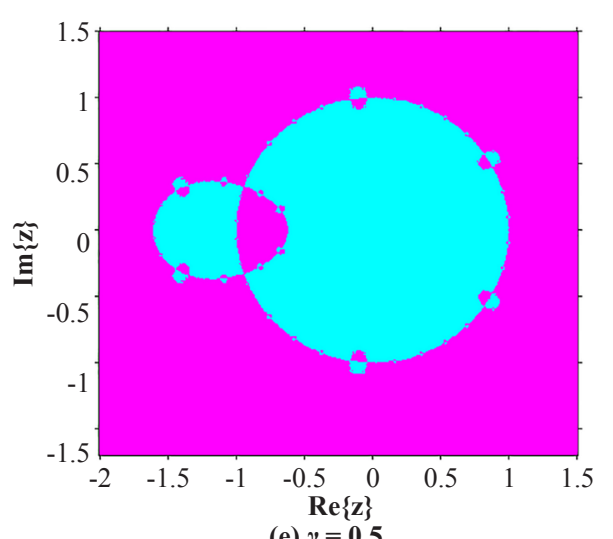

(e) $\gamma=0.5$

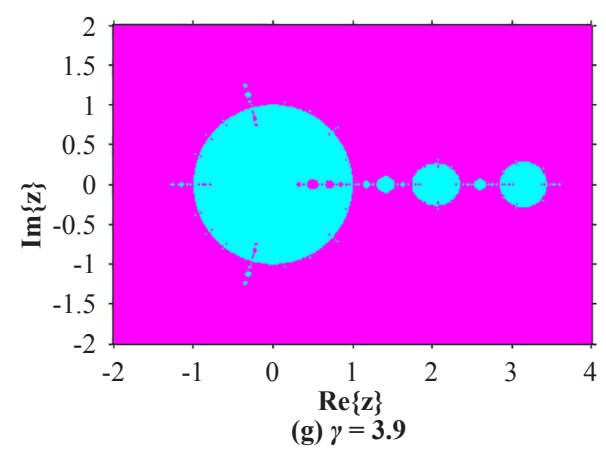

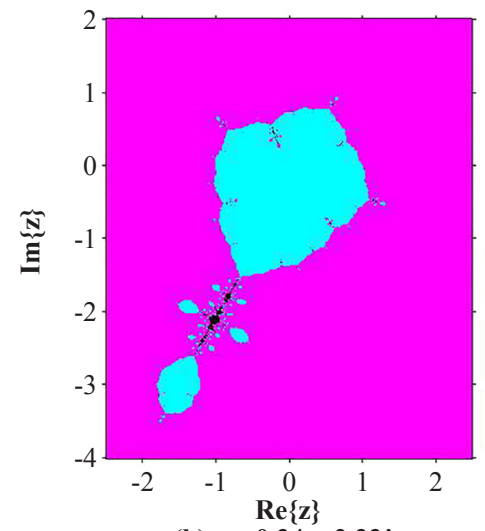

(b) $\gamma=0.34+2.33 i$

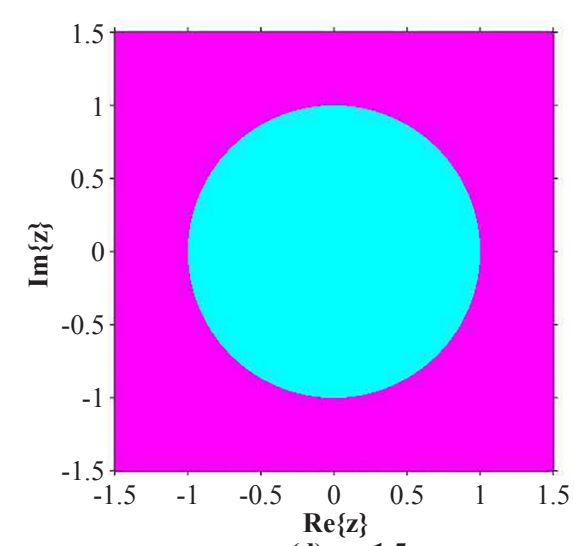

(d) $\gamma=\mathbf{1 . 5}$
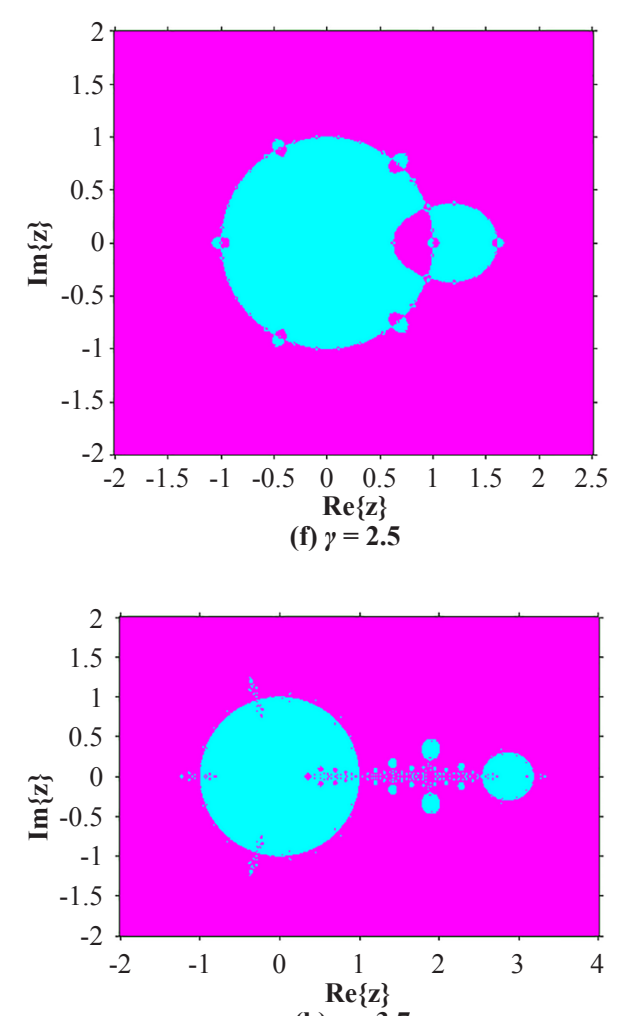

(h) $\gamma=3.7$

Figure 8. Dynamical planes 


\section{References}

[1] Maroju P., Magreñán Á. A., Motsa S. S., Sarría Í. Second derivative free sixth order continuation method for solving nonlinear equations with applications, subsection 8.5. J. Math. Chem. 2018; 56(7): 2099-2116.

[2] Parhi S. K., Gupta D. K. A sixth order method for nonlinear equations. Appl. Math. Comput. 2012; 218: 10548-10556.

[3] Ezquerro J., Hernández M. A. On Halley-type iteration with free second derivative. J. Comput. Appl. Math. 2004; 170: $455-459$

[4] Geum Y. H., Kim Y. I., Neta B. On developing a higher-order family of double-Newton methods with a bivariate weighting function. Appl. Math. Comput. 2015; 254: 277-290.

[5] Grau M., Diaz-Barrero J. L. An improvement of the Euler-Chebyshev iterative method. J. Math. Anal. Appl. 2006; 315: 1-7.

[6] Kou J., Li Y. A family of modified super-Halley methods with fourth-order convergence. Appl. Math. Comput. 2007; 189: 336-370.

[7] Ezquerro J., Hernández M. A. A uniparametric Halley-type iteration with free second derivative. Int. J. Pure Appl. Math. 2003; 6(1): 103-114.

[8] Kou J., Li Y., Wang X. A uniparametric Chebyshev-type method free from second derivatives. J. Appl. Math. Comput. 2006; 179: 296-300.

[9] Kou J., Li Y. On Chebyshev-type methods free from second derivative. Commun. Numer. Meth. Eng. 2007; 24: 12191225.

[10] Li D., Liu P., Kou J. An improvement of Chebyshev-Halley methods free from second derivative. Appl. Math. Comput. 2014; 235: 221-225.

[11] Traub J. F. Iterative methods for solution of equations. Murray Hill, New Jersey: Bell telephone laboratories incorporated; 1964.

[12] Amat S., Busquier S., Plaza S. Review of some iterative root-finding methods from a dynamical point of view. Sci. Ser. A: Math. Sci. 2004; 10: 3-35.

[13] Amat S., Argyros I. K., Busquier. S., Hernández-Verón M. A., Martínez E. On the local convergence study for an efficient k-step iterative method. J. Comput. Appl. Math. 2018; 343: 753-761.

[14] Argyros I. K., Cho Y. J., George S. Extending the convexity of nonlinear image of a ball appearing in optimization. Contemporary Mathematics. 2020; 1(4): 209-214. Available from: https://doi.org/10.37256/cm.142020405.

[15] Argyros I. K., George S. High convergence order q-step methods tions and systems of equations. Contemporary Mathematics. 2020; 1(3): 119-126. Available from: https://doi.org/10.37256/cm.132020403.

[16] Argyros I. K., Sharma D., Parhi S. K. On the local convergence of Weerakoon-Fernando method with $\omega$ continuity condition in Banach spaces. SeMA Journal. 2020; 77: 291-304. Available from: https://doi.org/10.1007/s40324-020$00217-\mathrm{y}$.

[17] Argyros I. K., Magrenan A. A. On the convergence of an optimal fourth-order family of methods and its dynamics. Appl. Math. Comput. 2015; 252(1): 336-346.

[18] Behl R., Cordero A., Motsa S. S., Torregrosa J. R., Kanwar V. An optimal fourth-order family of methods for multiple roots and its dynamics. Numer. Algor. 2016; 71: 775-796.

[19] Chicharro F., Cordero A., Torregrosa J. R. Drawing dynamical and parameters planes of iterative families and methods. Scientific World Journal. 2013; 2013: 780153. Available from: https://doi.org/10.1155/2013/780153.

[20] Cordero A., Torregrosa J. R., Vindel P. Dynamics of a family of Chebyshev-Halley type methods. Appl. Math. Comput. 2013; 219: 8568-8583.

[21] Cordero A., Guasp L., Torregrosa J. R. Choosing the most stable members of Kou's family of iterative methods. $J$. Comput. Appl. Math. 2018; 330: 759-769.

[22] Kou J., Li Y. An improvement of the Jarratt method. Appl. Math. Comput. 2007; 189: 1816-1821.

[23] Magrenán A. A. Different anomalies in a Jarratt family of iterative root-finding methods. Appl. Math. Comput. 2014; 233: 29-38.

[24] Singh M. K., Singh A. K. Variant of Newton's method using simpson's 3/8th rule. Int. J. Appl. Comput. Math. 2020; 6 : 20. Available from: https://doi.org/10.1007/s40819-020-0770-4.

[25] Neta B., Scott M., Chun C. Basins of attraction for several methods to find simple roots of nonlinear equations. Appl. Math. Comput. 2012; 218: 10548-10556.

[26] Petkovic M. S., Neta B., Petkovic L., Dzunic D. Multipoint methods for solving nonlinear equations. Amsterdam: Elsevier; 2013.

[27] Thukral R., Petkovic M. S. A family of three-point methods of optimal order for solving nonlinear equations. $J$. Comput. Appl. Math. 2010; 233: 2278-2284.

[28] Neta B. On a family of multipoint methods for non-linear equations. Int. J. Computer Math. 1981; 9: 353-361. 
[29] Sharma D., Parhi S. K. Local convergence and complex dynamics of a uni-parametric family of iterative schemes. Int. J. Appl. Comput. Math. 2020; 6(3): 83. Available from: https://doi.org/10.1007/s40819-020-00841-2.

[30] Sharma D., Parhi S. K. Complex dynamics of a sixth and seventh order family of root finding methods. SeMA J. 2020; 77(3): 339-349. Available from: https://doi.org/10.1007/s40324-020-00223-0.

[31] Sharma D., Parhi S. K. On the local convergence of a third-order iterative scheme in Banach spaces. Rendiconti del Circolo Matematico di Palermo Series 2. 2020. Available from: https://doi.org/10.1007/s12215-020-00500-x.

[32] Rahimian S. K., Jalali F., Seader J. D., White R. E. A new homotopy for seeking all real roots of a nonlinear equation. Computers and Chemical Engineering. 2011; 35: 403-411.

[33] Kou J., Li Y., Wang X. Fourth-order iterative methods free from second derivative. J. Appl. Math. Comput. 2007; 184: 880-885. 